Cinémas

Revue d'études cinématographiques

Journal of Film Studies

\title{
Deux pôles yang du nouveau cinéma chinois : Chen Kaige et Zhang Yimou
}

\section{Marie Claire Huot}

Volume 3, numéro 2-3, printemps 1993

Le nouveau cinéma chinois

URI : https://id.erudit.org/iderudit/1001194ar

DOI : https://doi.org/10.7202/1001194ar

Aller au sommaire du numéro

Éditeur(s)

Cinémas

ISSN

1181-6945 (imprimé)

1705-6500 (numérique)

Découvrir la revue

Citer cet article

Huot, M. C. (1993). Deux pôles yang du nouveau cinéma chinois : Chen Kaige et Zhang Yimou. Cinémas, 3(2-3), 103-125. https://doi.org/10.7202/1001194ar
Résumé de l'article

Seul deux cinéastes chinois sont connus aussi bien en Chine qu'à l'étranger. Il s'agit de Chen Kaige et de Zhang Yimou. L’article tente de démontrer la polarisation de leur cinéma respectif à partir de leurs positions qui sont diamétralement opposées. Celle de Chen est dite hanxu, allusive qui incite à l'auto-réflexion alors que celle de Zhang est du ressort yanggang, soit en faveur de l'émotion vive et directe. L'article rend compte de ces deux pôles dans leurs différences, mais aussi dans ce qui les unit, leur « impensé » qui touche à la différence sexuelle et à l'écriture chinoise. 


\title{
Deux pôles yang du nouveau cinéma chinois : Chen Kaige et Zhang Yimou
}

\section{Marie Claire Huot}

\section{RÉSUMÉ}

Seul deux cinéastes chinois sont connus aussi bien en Chine qu'à l'étranger. Il s'agit de Chen Kaige et de Zhang Yimou. L'article tente de démontrer la polarisation de leur cinéma respectif à partir de leurs positions qui sont diamétralement opposées. Celle de Chen est dite hanxu, allusive qui incite à l'auto-réflexion alors que celle de Zhang est du ressort yanggang, soit en faveur de l'émotion vive et directe. L'article rend compte de ces deux pôles dans leurs différences, mais aussi dans ce qui les unit, leur «impensé» qui touche à la différence sexuelle et à l'écriture chinoise.

\begin{abstract}
Two Chinese filmmakers, Chen Kaige and Zhang Yimou, are as well known abroad as they are in China. This article seeks to show how the polarisation between their respective approaches to filmmaking derives from the diametrically opposed positions they have adopted. Chen's position is called hanxu or allusive and favours self-reflection, while Zhang's is called yanggang and favours vivid, direct emotion. The article discusses the difference between these two polar opposites, but also explores their underlying unity, the "unthought" dimension, which incorporates sexual difference and Chinese writing.
\end{abstract}


Même s'il y a plusieurs cinéastes de marque, hommes et femmes, en République populaire de Chine, deux seuls sont bien connus tant chez eux qu'à l'étranger. Il s'agit de Chen Kaige, qui vit présentement aux États-Unis, et de Zhang Yimou, toujours en Chine ${ }^{1}$.

Tous deux ont reçu, en 1982, leur diplôme de l'Institut de cinéma de Beijing et appartiennent donc à la Cinquième génération de cinéastes. Leurs profils sont en plusieurs points semblables : même institution de départ et en même temps, même âge ou peu s'en faut, même sexe et mêmes circonstances historiques (en particulier la Révolution culturelle). En outre, Chen et Zhang ont fait ensemble deux films : Terre jaune (1984) et La grande parade (1985). Tous deux font un cinéma qui est critique de la tradition et de la culture chinoises, ainsi que du cinéma chinois qui s'est fait avant eux.

Malgré les similarités et coïncidences dans leurs cheminements respectifs, leurs positions sont aujourd'hui fort différentes, tant dans leur production que dans leur façon d'en parler. Cet article tente d'exposer ces deux modes de cinéma, deux pôles contrastants, en les comparant l'un à l'autre. Mon fil conducteur consiste en deux notions chinoises, le hanxu (mode implicite) et le yanggang (mode contrasté). L'un ou l'autre des positionnements entraîne une série d'effets esthétiques et idéologiques dont le plus important est, d'une part, la distanciation et de l'autre, la fascination qui se manifeste notamment par l'importance accordée soit à la nature, soit aux personnages, et l'insistance sur des objets de savouration ou de consommation.

Je ferai donc une incursion dans l'acception des deux termes chinois ci-haut mentionnés, dans l'usage particulier qu'en font Chen et Zhang, puis exposerai les techniques cinématographiques utilisées par chacun afin d'arriver à leurs fins respectives. Technique et thématique étant (ici) intimement liées, je passerai ensuite aux sujets de prédilection de ces deux cinéastes, aux objets et motifs qu'ils privilégient. J'espère ainsi montrer qu'il $\mathrm{y} a$ cheminement à l'intérieur même de l'œuvre de ces deux cinéastes, mais aussi cheminement vers deux modalités distinctes et tout autant valorisées dans la production culturelle chinoise actuelle, au moins dans celle réalisée par les hommes.

\section{Qu'est-ce que le hanxu et le yanggang?}

\section{Dans le discours de Chen et de Zhang}

Depuis la reconnaissance, en Euro-Amérique, de la Cinquième génération que l'on peut situer en 1988, avec l'attribution de l'Ours d'argent de Berlin au film Sorgho rouge de Zhang Yimou 
et l'attribution du Réveil-matin en or de Cannes au film Le Roi des enfants de Chen Kaige, j'ai été intriguée par la façon dont ces cinéastes parlaient de leurs propres œuvres. Un terme était récurrent chez chacun d'eux : le hanxu chez Chen Kaige et le yanggang chez Zhang Yimou.

\section{- «hanxu», le mode indirect de Chen Kaige}

Chen Kaige, parlant de son premier film Terre jaune, a affirmé: «La quintessence de notre style peut se résumer en un mot, "hanxu" 2 ». Je traduis ce mot par «indirect» ou «ambigu» parce que, à la lecture des nombreux interviews que Chen a accordés, en plus d'affirmer ce qu'il veut faire dans ses films, il insiste aussi sur ce qu'il ne veut pas faire : du cinéma noir sur blanc où tout est bien défini, clos, univoque et téléologique. Chen avoue avoir horreur de la facilité, du cinéma de divertissement comme le cinéma américain qui est, selon lui, «par trop clair ${ }^{3}$ » ou encore le cinéma chinois de la Révolution culturelle, qu'il dit «vide de sens ${ }^{4}$ ».

Chen est partisan d'un cinéma sérieux, c'est-à-dire qui traite de questions qui vont au-delà du ponctuel. C'est pourquoi il critique notamment les films de deux de ses confrères qui, d'après lui, s'enlisent dans l'ici-maintenant : L'Année de mon signe de Xie Fei, un film portant sur les problèmes actuels des jeunes à l'ère de l'économie de marché, n'est pas, à son avis, réussi. «Moi, j'aurais traité des problèmes éternels de la jeunesse ${ }^{5}$ », affirme-t-il. Judou de Zhang Yimou est également la proie de sa critique parce que ce film, d'après lui, expose les rapports vils et bas entre les hommes et les femmes 6 . Pour Chen, ce sont les rapports entre les êtres humains, dans leur humanité et dignité, qui importent plus que tout ${ }^{7}$.

Aussi peut-on constater que le terme hanxu implique une position qui est reliée à une volonté d'abstraction, de dépassement des circonstances particulières inscrites dans un temps donné, qui est à portée philosophique. Et, de fait, Chen décrit le travail de cinéaste comme étant une mission. Pour lui, ce n'est pas le cinéma lui-même qui importe, mais ce que peut provoquer le cinéma. Dans tous les interviews recensés, Chen parle de son devoir :

Je suis un membre de l'humanité (...) je me vois toujours comme un saint, je considère que dans mes films, je dois faire passer une idée (...). Au plus profond de moi, je me prends pour un éducateur (Aubert, p. 50).

Il parle aussi du devoir du spectateur :

Ce que la caméra nous invite à regarder n'est pas le paysage comme tel, ni le personnage comme tel, mais l'histoire de la civilisation chinoise; pour que l'on s'interroge sur les raisons qui font que cette ancienne nation et cette culture sont en retard par 


\section{— «yanggang», le mode direct de Zhang Yimou}

C'est à partir de son premier film Sorgho rouge que Zhang s'est mis à parler de sa position comme étant contre toute forme de hanxu et en faveur du yanggang : "Je n'ai rien à voir avec le "hanxu", avec une façon contournée de faire»; «Les personnages (...) sont simples et heureux (...); je ne les représente pas de manière "hanxu", désincarnée : ils n'ont pas de problème» $(\mathrm{Li}, \mathrm{p}$. $44)$; «Ce que je veux créer c'est un beau "yanggang", fort / viril» (Zhong).

À chaque fois que Zhang Yimou discute de ses films, il parle à la fois d'effet esthétique et psychologique. Ce qui semble préoccuper Zhang, c'est l'effet émotif de ses films sur l'auditoire, un effet créé par une esthétique foudroyante et par des personnages forts, le tout haut en couleurs. Zhang ne discute pas en termes politicophilosophiques. Même si Zhang continue à nier toute allusion à la politique contemporaine chinoise dans ses films, de nombreuses lectures ont été faites en ce sens. En fait, Zhang affirme ne se baser que sur ses propres expériences :

Ce que je peux dire c'est que, c'est ainsi que je vois la vie, que je vois le destin en ce moment. Comment ça changera dans le futur, je ne le sais pas (Chua, p. 30).

Je n'ai pas tant de choses à dire.(...) Un film ne devrait pas être comme une conférence philosophique (Zhang Yimou, p. 54).

Plusieurs anecdotes illustrent l'audace de Zhang, que ce soit sa volonté de faire pousser en période de sécheresse un immense champ de sorgho; ou encore d'obtenir, sous l'opprobre populaire, un divorce afin de marier la vedette de tous ses films, Gong Li. Le commentaire suivant, du directeur artistique de son dernier film, Qiuju, une femme chinoise, ne peut qu'être lu ironiquement : «Zhang est démocratique (...). Ce qu'il dit, c'est ce que l'on fait. Il a le dernier mot» (Pan, p. 33). Zhang est certes opiniâtre, comme ses personnages féminins. On peut dire qu'il reprend, en la transposant dans une autre période, sa propre vie ${ }^{9}$. Tout récemment, il affirmait que «survivre, c'est gagner» (Pan, p. 38). Zhang survit en Chine même, où ses films Judou et Épouses et concubines ont été interdits jusqu'en août 1992. Que ce soit à cause des références sexuelles ou politiques ou trop éminemment personnelles (donc bourgeoises), il n'en demeure pas moins vrai que Zhang choque, provoque, mais gagne. 
Concluons le portrait des deux cinéastes par ces propos d'un critique chinois qui sont typiques de leur présentation habituelle :

Chen ressemble à un lettré profond (...) il a la sobriété et la profondeur du philosophe. (...) Zhang est devenu un personnage rappelant les contes populaires; il ressemble à un ouvrier ou à un paysan ordinaire (Hua, pp. 43 et 68 ).

\section{Le «hanxu» et le "yanggang» dans la culture chinoise}

\section{- le «hanxu» comme mode traditionnel dominant}

Il est très facile de retrouver de nombreuses références au hanxu dans les textes traditionnels chinois. En effet, l'on peut dire que c'est un des termes clés de l'esthétique chinoise traditionnelle. En poésie, sa première occurrence, il se traduit par «allusion» ou encore «évocation»; le poète, dans les images, dans les mots, choisit des expressions qui ne font du sens que lorsque le lecteur les associe à d'autres mots et à sa propre expérience de la nature et du monde en général. C'est donc une préférence pour l'ellipse, pour le non-dit, le suggéré. En d'autres mots, cette poésie chinoise se lit entre les mots / images, pour un sens qui les transcende.

Cette même prédilection se retrouve dans la calligraphie et dans la peinture où elle se manifeste par la valorisation des blancs du support, que l'écriture / peinture sert à mettre en relief. C'est la place laissée à l'imagination qui prime. Aussi, de telles peintures ont-elles un répertoire limité (comme la poésie, d'ailleurs) et sont surtout constituées d'un minimum de traits, de peu de couleurs. Cela est dit «fade» (pingdan 10 ) et est donc de nature suggestive, puisque la matière peinte ou écrite sert de tremplin au spectateur, lui permet d'aller au-delà de cette matière. Tous les arts, dans cette optique, ont le monde naturel comme objet et l'introspection comme objectif.

L'idéal est l'harmonisation de l'œuvre à l'homme, puis de l'homme au monde. C'est cette vision éthique de l'œuvre d'art qui a primé en Chine. L'harmonie signifie qu'il n'y a pas d'excès (autobio)graphique, ni d'émotions fortement inscrites dans l'œuvre. Cela est exprimé en chinois par l'expression quasi millénaire : «Le beau du yang ferme et du yin flexible», «yanggang yinrou zhi mei». Étant donné qu'une participation active du lecteur / spectateur est sans cesse requise, on peut parler à juste titre, je crois, de ces formes d'art hanxu comme des filtres, ou encore des codes qui nécessitent au préalable des connaissances et reconnaissances de plusieurs ordres (tel que d'avoir parcouru dix mille lis ou d'avoir lu dix mille livres).

Ce sont ces arts nobles de la Chine impériale - poésie, peinture et calligraphie - , appelés d'ailleurs les «trois perfections» 
(sanjue) qui ont commandé l'unique vision artistique chinoise, très fortement liée à la philosophie, à la cosmologie, grosso modo jusqu'au XXe siècle. L'homme a sa place dans le cosmos, mais ce n'est pas en son centre; il participe harmonieusement au fonctionnement, s'il est intégré aux rapports sociaux convenables et s'il est en équilibre avec l'environnement naturel.

\section{- le yanggang comme nouveau mode d'expression}

Si l'on retourne à l'expression «le beau du yang ferme et du yin flexible», «yanggang yinrou zhi mei», on constate que le terme yanggang est le même que la première partie de l'expression entière. En termes purement logiques, le yanggang serait un aspect de cette conception du beau / bon, de cette harmonisation et, employé seul, impliquerait un déséquilibre entre les pôles yang et yin. En effet, il ne servirait à rien - je l'ai tout de même fait pour m'en assurer - de chercher ce terme yanggang dans les dictionnaires même les plus spécialisés : il ne s'y retrouve que comme une moitié de l'ensemble harmonieux ${ }^{11}$.

Que signifie-t-il donc au juste? On se rappellera que Zhang Yimou n'emploie pas de termes philosophiques pour décrire son entreprise. En fait, yanggang est un néologisme qui a émergé dans les années 80 en Chine, dans au moins trois formes particulières d'art, à ma connaissance : au cinéma, en littérature et dans les arts plastiques. Il se veut tout le contraire de l'harmonie, de l'équilibre plat, du discret; c'est une notion qui embrasse à la fois le travailleur des arts, sa production et l'effet sur le spectateur. On peut le traduire tantôt par force virile, cran, impétuosité, émotion et expression vives, le primitif, bref, par toute manifestation directe, sensorielle.

Le mode yanggang se situe donc aux antipodes du hanxu et, par conséquent, à l'encontre de la tradition chinoise mais aussi des prescriptions de la Chine actuelle. Comme expression artistique, il ne nécessite pas de décodage particulièrement savant; ni la compréhension d'un contexte autre que celui qui est immédiat. Les seuls antécédents possibles se trouveraient dans la culture populaire des contes, arts martiaux, peintures du nouvel an, et autres manifestations traditionnellement non incluses dans les arts nobles. Avant tout, l'appréhension est directe parce que la représentation touche aux cordes sensibles. Le yanggang veut ébranler, provoquer par des formes imagées, veut faire ressortir le refoulé. Il faut ajouter ici que cette nouvelle vague est, quoique très populaire, exclusivement un mode masculin. 


\section{- implications de l'emploi de ces termes aujourd'hui}

Qu'il y ait conscience ou non des connotations des termes hanxu et yanggang, respectivement chez Chen et Zhang, n'atténue en rien le fait que le premier terme est lourdement imprégné de la tradition philosophico-esthétique chinoise et que l'autre est, si l'on peut dire, un signe des temps qui n'est pas encore tout à fait conceptualisé. On pourrait m'objecter qu'après tout, un Chinois s'exprime en chinois avec des termes qui sont déjà connotés, qu'il ne peut pas davantage s'en sortir que quiconque dans toute langue. Je répondrais à cela que, d'abord, le terme hanxu, depuis le XXe siècle, a été traîné dans la boue par plus d'un, et non parmi les moindres, par Lu Xun qui disait : «Mieux vaut ne pas utiliser le mode "hanxu"; les gens ainsi seraient confus» (Hanyu da cidian, p. 230). Après l'avènement de la Chine nouvelle, Mao et ses sbires ont mené une attaque sans merci aux utilisateurs de techniques hanxu, qui laissaient trop de place à l'interprétation. Aussi, que Chen réutilise ce terme dans les années 80 n'est nullement innocent. En quelque façon, il y a une allégeance à la culture traditionnelle chinoise; et en plus une réaction, une position allant délibérément à l'encontre du réalisme socialiste (qui, lui, est noir sur blanc).

Quant à l'utilisation par Zhang Yimou de yanggang, on peut $\mathrm{y}$ lire un refus plus global du fait chinois qui rejoint le parti pris de nombreux jeunes Chinois d'aujourdhui. Ce rejet se signale par une fascination tant pour un certain Japon et une certaine Amérique, devenant les représentants de la «libre expression», d'un mode débridé. Il y a plus d'un lien entre le cinéma japonais et Zhang Yimou. C'est le Japon qui a le premier reconnu Zhang, en lui décernant, en 1987, pour son jeu dans Le vieux puits, le prix de la meilleure interprétation masculine au Deuxième Festival international de Tokyo. C'était la première fois qu'un Chinois recevait un tel prix à l'étranger (Hua, p. 77). En outre, ses propres films ont des affinités avec des films japonais de la nouvelle vague qui présentent des samouraïs primitifs ou encore des rapports sexuels entre hommes et femmes de façon naturaliste. Zhang se serait d'ailleurs inspiré du film Onibaba (Les Tueuses) de Shindo Kaneto pour réaliser Sorgho rouge ${ }^{12}$.

Le yanggang n'a donc pas de tradition indigène en Chine. Lorsqu'un jeune critique de la peinture d'avant-garde utilise ce terme, il l'associe à l'Occident où l'on se positionne contre la tradition, où l'on ne recherche pas l'harmonie (Zhou, pp. 132134). Ce terme est donc un néologisme en Chine, un terme qui représente dans toutes les formes d'art une ouverture sur, sinon le radicalement nouveau, au moins le refoulé de la culture chinoise. 
Une chose est certaine : l'usage aujourd'hui de l'un ou l'autre de ces termes pour décrire une démarche artistique dénote un rejet des fondements idéologiques et artistiques de la Chine socialiste ainsi qu'une rupture avec tout le passé. Chen réinterprète les fondements classiques en y mettant au centre l'individu; Zhang préconise l'hétérodoxe et pose la centralité du moi dans la production artistique. Ces deux démarches sont également des dénonciations de forclusions diverses dans la production culturelle chinoise dans son ensemble.

Une autre chose est certaine : les deux expressions occultent la différence sexuelle. Le hanxu serait un positionnement qui est censé englober toute l'humanité mais qui, en fin de compte, ne vaut que pour la moitié, celle des hommes. Un parallèle peut être fait avec l'emprunt d'une voix féminine par un poète masculin dans la poésie classique. Ou encore, dans les écrits daoïstes, de la valorisation du principe féminin non pas pour valoriser celui-ci en soi, mais pour suggérer un principe masculin autre. Depuis toujours, le hanxu n'a jamais été un mode d'expression des femmes, mais bien des hommes. Chen, en ce sens, ne révolutionne rien. Privilégier le yin, le versant sombre, «féminin», n'est qu'un aspect du yang. Le soi-disant équilibre du yin et du yang n'est toujours qu'un yang redoublé.

Avec son utilisation du yanggang, Zhang est facilement taxable de machisme. Son mode d'expression fort, viril s'allie à une présentation de la (belle) femme comme objet. Ce qu'il nous présente comme refoulé, le désir dans toutes ses manifestations, est pour l'homme dans son sens restreint, par opposition à la femme.

Une écrivaine chinoise a discuté du yanggang comme phénomène courant chez les écrivains chinois d'aujourd'hui (Can Xue, pp. 332-351). Le ton de son texte est extrêmement moqueur. Elle joint en une expression le souffle yanggang et la masculinité et $\mathrm{y}$ associe toute une série très comique d'épithètes comme «force primitive», «ardeur héroïque»; elle décrit ses représentants au souffle rauque et au sang chaud, comme vivant dans une chaumière, dans la nature, en pleine forêt et avec de jolies femmes aux pieds bandés. Ils ne sortent de là que si une voiture officielle vient les chercher pour les emmener à l'étranger. Sous sa plume, ces «camarades d'armes» ont une libido sans pareille qui fait que des filles ennuyantes, venant d'une petite ville sans importance et n'ayant pas les pieds bandés, n'ont aucune chance de les attirer. En matière de littérature, ce sont eux - et non pas elle - qui ont un sens de la mission et un sens historique, ainsi que l'esprit national. Ne peut-on pas y reconnaître un peu Chen Kaige et Zhang Yimou? 
L'usage fréquent de la première personne du pluriel chez Chen et de la première personne du singulier chez Zhang est une autre indication non seulement de leurs visions opposées mais encore de leurs interlocuteurs supputés. Chen s'adresse à une communauté (d'intellectuels), et se penche sur des questions universelles; Zhang parle de lui-même, mais libre à tout autre (homme) de s'identifier. L'une comme l'autre des positions est exagérée : Chen se prendrait-il pour le sauveur du monde? Une analogie avec le lettré / éducateur d'antan se servant des arts pour communiquer la bonne voie - wenyi zai dao - n'est pas farfelue ici. Quant aux affirmations désinvoltes de Zhang, elles pêchent aussi par l'autre excès, celui d'un art pour l'art, d'un je-m'en-foutisme forcené. Après tout, Zhang vit en Chine communiste où une telle «inflation du moi» - ziwo pengzhang — n'est pas permise. Et Chen vit aux Etats-Unis, où les préoccupations humanistes d'une telle envergure sont déplacées.

\section{Cadre naturel et cadre humain}

La polarisation yang, désignée par hanxu et yanggang, est manifeste à l'examen des films de Chen et de Zhang. Un cinéma hanxu ne se préoccupera ni de l'histoire à raconter, ni de l'illusion de personnages réels; il sera fortement allusif, symbolique et laissera une grande part (visuelle et sonore) à la nature, afin de faciliter l'introspection. C'est en quelques mots la matrice du cinéma de Chen Kaige. Quant au cinéma yanggang, les personnages seront centraux, et leur psychologie sondée; tout concourra à provoquer, que ce soit par l'exposition de tabous, des rapports intimes entre hommes et femmes, d'objets et pratiques exotiques, par la mise en scène d'effets sensoriels contrastés. Ce sont bien les caractéristiques du cinéma de Zhang Yimou.

À travers ses films, Chen Kaige laisse une place de plus en plus prédominante au paysage, lui accordant ainsi un statut plus important, d'une certaine manière, que celui des personnages. Dans son film La Vie sur un fil, ce qui marque le spectateur, voire l'éblouit, ce sont les perspectives grandioses, les spectaculaires cascades d'eau, les immenses plateaux, bref un paysage dramatique souvent exhibé en plongée, dans de lents panoramiques et sans personnage. Le paysage devient la projection de l'âme collective du peuple, peut-on dire, et le lieu de la résolution des contradictions, des tensions (aussi minimes soient-elles).

C'est l'amenuisement progressif de l'espace qui se produit dans le cinéma de Zhang Yimou, en faveur d'une architecture humaine devenant de plus en plus contraignante. Dans Épouses et concubines, il n'y a aucune prise de vue de paysages; la majorité des scènes se déroule à l'intérieur de la maison d'un riche, maison 
rigidement symétrique et d'un extérieur uniformément gris. Ce qui frappe le spectateur, c'est le contraste entre cette morne régularité et les personnages féminins, hauts en couleurs, qui y sont emprisonnés. Le drame d'une intensité extrême a lieu à huis clos.

\section{Le cadre naturel chez Chen : le film peinture}

Dans les films de Chen, s'il y a héros, c'est la nature. L'humain représenté n'est, somme toute, pas important. C'est celui qui regarde qui l'est. L'écran est un miroir. Aussi, la photographie encadre-t-elle les humains d'une manière qui les décentre. La majorité des plans du Roi des enfants présente, par exemple, de façon tronquée le personnage La Perche : on ne lui voit pas la tête lorsqu'il arrive la première fois à sa salle de classe. Ou encore, il est complètement à l'extérieur du cadre, comme dans la scène du début où, sur le seuil, il converse (si on peut désigner par «conversation» les quelques mots échangés, entre la cigarette et l'allumette). Ou bien, et c'est sans doute le mode de représentation le plus utilisé par Chen, le personnage est perdu dans un paysage où, parce qu'il fait noir ou bien parce que celui-là est trop loin, il est flou, à peine perceptible, tout comme dans la peinture de paysage chinoise, la peinture de prédilection des lettrés, où l'homme est représenté conformément à son importance dans le cosmos, à savoir infiniment petit. Terre jaune et La Vie sur un fil offrent plusieurs scènes où les personnages, filmés en plan général, sont perchés sur une hauteur : la famille paysanne et le soldat prenant, en silence, un maigre repas de bouillie; le vieil aveugle, assis seul en haut du plateau, jouant son qin, «pour lui-même» (pour la nature, pour les spectateurs).

Ces scènes sont évocatrices à plus d'un titre de la noblesse du peuple chinois : sa grandeur dans la simplicité, la beauté physique de sa terre natale. Ces scènes se lisent comme les peintures traditionnelles : dans le recueillement, le spectateur tente de s'unir en esprit à ce sage à peine esquissé qui, lui, vit en harmonie avec la nature. Aucune spécificité matérielle (un clair-obscur, peu de couleurs et de traits, peu d'attraits sensoriels) n'intervient pour empêcher l'association. En fait, le cinéma de Chen comporte les trois points majeurs du cinéma inspiré de l'esthétique traditionnelle chinoise : les prises de vue de très haut et les séquences «rouleaux»; le cadrage asymétrique; la continuité dans le mouvement ${ }^{14}$. L'introspection est paradoxalement possible grâce à la distanciation. 


\section{Cadre humain chez Zhang : le film photo-roman}

Les films de Zhang sont au contraire fortement ancrés dans une matérialité séduisante, dans un empire des sens. Les personnages, comme les décors et les musiques, ont des contours bien délimités. On ne s'identifie pas nécessairement à ces personnages, mais on est captivé par eux, par leur quête d'assouvissement. Ses personnages sont au centre de l'écran et ils nous envoûtent. Épouses et concubines commence presque de la même façon que Sorgho rouge : avec le visage du personnage féminin, en gros plan, en plein centre de l'écran, sans aucun arrière-plan. Au début de Sorgho rouge, le narrateur d'âge moyen nous annonce, avec un effet comique certain, que ce qui suit est «l'histoire de mon grandpère et de ma grand-mère». Or la première prise de vue présente cette future grand-mère - que l'on ne verra jamais vieillir - au moment de son mariage : une magnifique jeune femme. Il y a là un clin d'œil concupiscent à l'auditoire. On est à au moins 10,000 lis de la peinture chinoise de paysage. Dans Épouses et concubines, on regarde, admire, scrute - comme si on regardait une photographie, la caméra étant absolument statique - le visage d'une femme, au sujet de laquelle tout nous est encore inconnu. Elle nous regarde mais sans nous regarder, puisqu'elle est en conversation avec sa belle-mère, en hors champ. Mais elle est l'objet de notre regard. Dans Judou, elle se prête même au voyeurisme de Tianqing. Dans tous les films de Zhang, ce qui nous est donné à voir sont les femmes, leurs regards, leurs corps aussi, qui deviennent nos objets, pour notre plaisir de voyeur. L'environnement, qu'il soit naturel ou architectural, le décor et les costumes servent ici à donner corps à l'émotion esthétique, indissociable du récit de la vie (horrible) de ces (belles) femmes. Le comble du contraste est créé dans Epouses et concubines par l'opposition entre les plans fixes, en noir et blanc, de la résidence austère et les plans sur les corps voluptueux des femmes, habillées de couleurs voyantes et de bijoux rutilants.

Si les films de Chen se rapprochent de l'esthétique de la placide peinture des lettrés, ceux de Zhang se rapprochent alors davantage des genres mineurs, à commencer par les peintures décoratives de belles femmes, un genre deux fois mineur parce qu'appartenant à cette catégorie peu appréciée, l'art du portrait et parce que ne représentant pas des personnages illustres (ce qui est presque exclusivement réservé aux hommes), mais de jolis minois anonymes. On pourrait également inclure dans la comparaison la photographie ayant comme sujet / objet des êtres humains, cet art non indigène si indigeste pour une culture méprisant le mimétisme; ou encore les peintures du nouvel an où figurent des 
bébés, des poissons, des cornes d'abondance, en chaudes couleurs vives; ou même les illustrations érotiques, un des plus grands tabous de la Chine tout au moins actuelle. Toutes ces formes d'expression populaire sont des produits de consommation. Ils servent à décorer et / ou à titiller. Avant tout, ils sont fascinants.

\section{La savouration et la consommation}

Suivant la logique des paramètres hanxu et yanggang, il est évident que les sujets et objets de prédilection de chaque cinéaste seront nécessairement fort différents. Chez Chen, c'est un objetmétaphore qui facilite l'autoréflexion : le miroir. Il est un intermédiaire pour la savouration. Chez Zhang, c'est l'objet de consommation par excellence : la nourriture. Celle-ci permet l'échange, le partage et le départage.

\section{La savouration chez Chen}

«hanxu»: ni avalé ni recraché (Hanyu da cidian, p. 230).

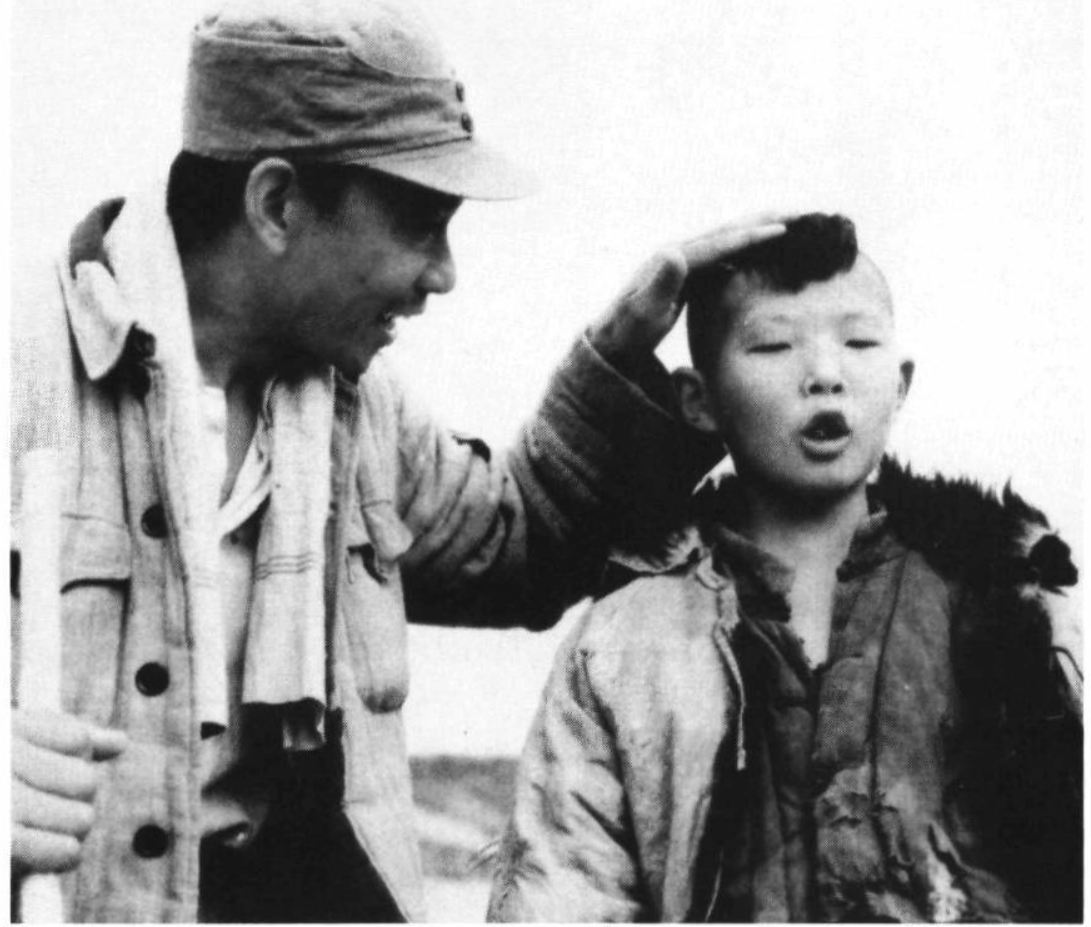

Terre jaune de Chen Kaige (1984) 
Chez Chen, les personnages sont à peine esquissés. Dans chaque film, on retrouve au moins deux générations d'hommes qui soulèvent la question de la transmission du savoir et de la connaissance. De plus, presque tous les protagonistes, par ailleurs de sexe masculin, ont un handicap physique. Un des personnages, dans La grande parade, a les jambes arquées; il essaie d'y remédier en se torturant la nuit avec des tiges de métal pour les redresser. Cela évoque le désir de conformisme à tout prix chez l'être humain; le mutisme des vieux, dans Terre jaune et Le Roi des enfants, symbolise leur incapacité à s'adapter au monde contemporain, à le comprendre, à parler le même langage - ils savent, en revanche, chanter. Quant aux aveugles de La Vie sur un fil, leur cécité est l'envers de la métaphore, car ce sont eux - et les spectateurs - qui voient : le suicide de la fille amoureuse du plus jeune aveugle est «visible» par lui seulement parce qu'il le perçoit et parce qu'elle le fait pour lui, devant lui. L'intérêt de ce dernier film réside dans la position dans laquelle est mis le spectateur qui voit tout, y compris la façon de «voir» des aveugles.

Étant donné que ces personnages «marqués» sont les plus valorisés, ceci suggère que la déficience sensorielle permet d'accéder à un niveau plus élevé de connaissance. Que Chen ait affirmé qu'il n'a pas de préférence pour l'un ou l'autre des deux aveugles de La Vie sur un fil, qu'il les affectionne tous les deux (Schell), n'étonne pas : le vieux et le jeune forment ensemble un archétype de l'être humain, divisé entre la foi (aveugle!), la libido et l'autodiscipline.

En d'autres mots, la réflexion que Chen offre sur la condition humaine se fait à partir de personnages masculins désincarnés, censés représenter l'humanité. Dans les moments forts, qui sont dans les films de Chen souvent non diégétiques, où l'on ne parle pas mais où l'on entend des bruits ou chants hors champ, on voit soit un homme, soit la nature. Je ne donnerai qu'un exemple qui me semble représentatif des deux choix. Il est tiré du Roi des enfants : la scène ouvre sur un paysage, au crépuscule, en plan général; on y voit, contre un ciel nuageux, un arbre et une grosse roche; dans le plan qui suit, cette roche bouge et s'avère être le personnage La Perche qui lentement s'étire, puis balance, comme un épouvantail au vent, les manches non enfilées de sa veste.

Cette scène, comme tant d'autres, permet de nombreuses interprétations. Le spectateur doit l'investir de quelque chose provenant de lui-même, car un temps aussi lent, où rien n'est raconté, où le personnage même n'est plus personnifié, l'interpelle dans sa propre vie. Le spectateur est donc débranché de l'histoire, en l'occurrence de La Perche. Dans La Vie sur un fil, cette 
incitation à l'autoréflexion est encore plus poussée à cause des chansons hors champ, aux thèmes largement humanistes, comme «À quand l'homme?», «Quand deviendrons-nous frères?», qui sont entendues alors que s'étalent devant nous des scènes de bataille. Que l'un ou l'autre des camps gagne la bataille, ou que ce soit - plus incroyable encore! - le vieil aveugle qui, par sa musique, l'arrête, n'a pas d'importance : on sait que l'on est en train de regarder, de savourer un (beau) film poéticophilosophique.

S'il y a objets insistants dans les films de Chen, ils ne sauraient être vulgaires, terre à terre. En effet, ceux-ci sont davantage du registre culturel chinois traditionnellement valorisé : la cithare qin, la musique chinoise en général, le cerf-volant, le miroir, le jeu d'échecs, l'écriture et puis le miroir. Ces objets sont tous fortement connotés. Dans La Vie sur un fil, le qin joué par les deux aveugles (même si on ne les voit jamais jouer!) représente la pureté; c'est le jeu musical du vieil aveugle qui rompt les batailles entre clans; c'est par la discipline dans le jeu que l'aveugle peut vivre. La musique fait traditionnellement partie des arts nobles en Chine, et la cithare est un des instruments les plus prisés. C'est aussi le cas des échecs, un autre jeu de lettrés. Dans Le Roi des enfants, il y un plan fixe qui nous montre des jeunes en train de jouer aux échecs (cette scène suit une scène de repas communautaire, mais est beaucoup plus importante en terme de temps et donc de valeur); on y voit aussi un La Perche perdu dans ses pensées. Musique et jeu d'échecs offrent des pauses pour la contemplation, pour l'autoréflexivité.

Cependant, c'est le miroir qui revient dans les films de Chen, dans La grande parade, Le Roi des enfants, La Vie sur un fil. Le miroir est sans doute l'objet de contemplation le plus évident pour Chinois et non-Chinois. Étant une métaphore universelle, il s'inscrit parfaitement comme choix de prédilection d'un cinéma hanxu. Dans La grande parade, un jeune garçon sort fréquemment son miroir quand il est seul pour s'assurer (ou se rassurer?) de son allure. Il est en pleine période de formation d'identité; aussi, son passage au nivellement anonyme d'un camp d'entraînement est-il encore plus un choc pour lui que pour un adulte. Dans Le Roi des enfants, dans un autre moment où «rien» ne se passe, La Perche se scrute la nuit dans un miroir, qui en plus dédouble son image puisqu'il est brisé au milieu. Le spectateur regarde, là aussi, un homme en train de se regarder et de s'examiner. Dans La Vie sur un fil, le vieil homme porte sur lui un miroir que lui aurait légué sa mère; qu'un non-voyant ait un miroir suggère, bien sûr, que l'objet a une fonction autre qu'utilitaire. Le miroir sert dans ces 
deux derniers films de sonde pour l'âme. En geste de réconciliation, le vieil aveugle offre son miroir à la jeune fille amoureuse de son disciple. Mais là encore, le miroir outrepasse sa fonction première : le spectateur voit, certes, la jeune fille s'y mirer, mais il voit encore le jeune aveugle tenir cet objet pour celle-ci, comme si le miroir, c'était lui. Pendant cette scène étrange, le jeune dit : «Je ne vois / perçois pas» (wo kan bu jian). Lorsque, tout de suite après, la fille se suicide, le miroir tombe. Cet objet a un statut de talisman. Cette interprétation se justifie par le fait que les deux aveugles, par leur seule présence comme par leur musique, sont les miroirs de la société environnante. Est-ce que le film même ne serait pas voulu comme un miroir réfléchissant notre image, ce que nous, spectateurs, sommes? Un miroir permet de voir de soi ce que l'on veut y voir. Il n'est jamais un calque parfait. Il y a toujours un écran, un filtre.

Le miroir fournit chez Chen une «matière» pour la réflexion. C'est le point de départ pour une quête de soi, du sens de la vie, de l'univers. Aussi n'y a-t-il jamais de regard direct, de transmission ou d'échange dans son cinéma. Les yeux sont tournés vers l'intérieur.

\section{La consommation chez Zhang}

En République populaire de Chine, où le puritanisme est de rigueur, la résistance la plus vigoureuse à l'ascétisme prôné par l'État ne s'est pas rencontrée dans les domaines de la corruption, du contrôle des naissances, ou dans le sexe, mais dans les banquets (Anderson, p. 200).

Alors que les films de Chen nous éloignent de plus en plus d'un récit à suivre, sur lequel on puisse se «suspendre», les films de Zhang nous happent, nous entraînent totalement dans la vie de leurs personnages, dans leur histoire. Ce n'est pas que les films de Zhang soient, comme beaucoup des films de Chine et d'ailleurs, inféodés à l'histoire à raconter; c'est parce que tout - sons et images, sur l'écran ou hors champ - concourt à créer une tension, un (trop-)plein d'émotions.

Tous les films de Zhang Yimou ont des personnages féminins comme protagonistes, et ceux-ci prennent, d'un film à un autre, de plus en plus d'importance, au détriment des personnages masculins. Cependant, la vie de ces femmes est totalement axée sur leurs rapports avec les hommes. En effet, tous les films de Zhang jusqu'à présent traitent des rapports entre les hommes et les femmes, et tout particulièrement des rapports sexuels entre les deux sexes. Le monde représenté est un monde sexiste où les femmes se débattent afin de pouvoir non seulement survivre, mais 
vivre selon leur désir. Les personnages féminins sont tous dotés de désirs très forts : dans Sorgho rouge, de choisir son époux, de venger son ancien amant; dans Judou, de se trouver un partenaire sexuel, de quitter la teinturerie et le clan; dans Epouses et concubines, de coucher avec l'époux, afin de diriger la maisonnée; dans Quiju, une femme chinoise, d'être moralement indemnisée pour la possible stérilité de son époux. Les hommes servent de support pour dévoiler les agissements des femmes. Lorsque Judou et Tianqing font l'amour ensemble, on ne voit pas le visage de l'homme, mais celui de la femme. Il en est de même dans Sorgho rouge. Dans Épouses et concubines, on ne voit même pas de près l'ennemi commun, le mari. Et pourtant, s'il est vrai que «dans un film, ce que l'on ne voit pas, c'est ce qui importe le plus ${ }^{15}$ », alors les films de Zhang sont au sujet des hommes, du sexe masculin. Aussi, que Zhang ait dit d' Épouses et concubines : «C'est peutêtre au sujet des femmes, et puis, peut-être pas 16 » ne surprend pas. Il s'agit bien de traiter des hommes, et ce, non pas de façon abstraite, dans leurs relations entre eux, mais spécifiquement dans leurs relations sexuelles avec les femmes. Zhang brise là un tabou, non seulement par ce sujet délicat, mais par l'assignation de rôles peu flatteurs aux hommes. Si l'on prend Judou en exemple, le personnage de Tianqing, l'amant de Judou, ne vaut pas plus que le vieil impuissant qui la torture, ou encore que son fils qui la séquestre. Tianqing, timoré, est incapable de s'assumer, ni de prendre position. Il ne peut que regarder misérablement Judou avorter; il ne sait quoi lui dire. Il est comme un mur.

Zhang nous donne donc sa vision du patriarcat, un monde où les hommes abusent de leur pouvoir sur les femmes, plus particulièrement dans leurs rapports sexuels avec elles. C'est le mode / monde de la consommation. Bien qu'il y ait profusion de détails visuels et sonores dans ses films, des choses merveilleusement exotiques (telles que l'amour dans les champs de sorgho, le massage des pieds féminins) qui leur confèrent une matérialité excessive, luxuriante, c'est la nourriture qui est avant tout emblématique du pouvoir patriarcal.

Dans Sorgho rouge, les héros vivent bien parce qu'ils ont pris le contrôle de la distillerie d'alcool de sorgho. On notera néanmoins que même si c'est la femme qui en est propriétaire, c'est vite son conjoint qui non seulement trouvera la bonne recette (uriner dans l'alcool), mais encore qui gérera l'entreprise. Dans Judou et dans Épouses et concubines, quiconque détient le pouvoir a simultanément le contrôle sur la nourriture. Judou et Tianqing ne s'évadent pas du domaine du vieil impuissant en grande partie parce c'est là où ils trouvent à manger. À chaque fois que Judou 
l'implore de quitter ce lieu insupportable, Tianqing rétorque: «Mais où est-ce qu'on irait? Qu'est-ce qu'on mangerait?» Épouses et concubines fonctionne de façon similaire. La vie des femmes est régie par quelques règles sévères mais fort simples, dont l'une est de manger en commun, dans une salle nue, à l'exception d'immenses portraits d'ancêtres suspendus aux murs. Celle des quatre qui a accueilli le mari la nuit précédente choisit le menu du jour. Ce qui est au départ non comestible le devient et ce qui est comestible devient immatériel. La nourriture (et l'alcool) sont, dans les films de Zhang, les objets les plus éthérés.

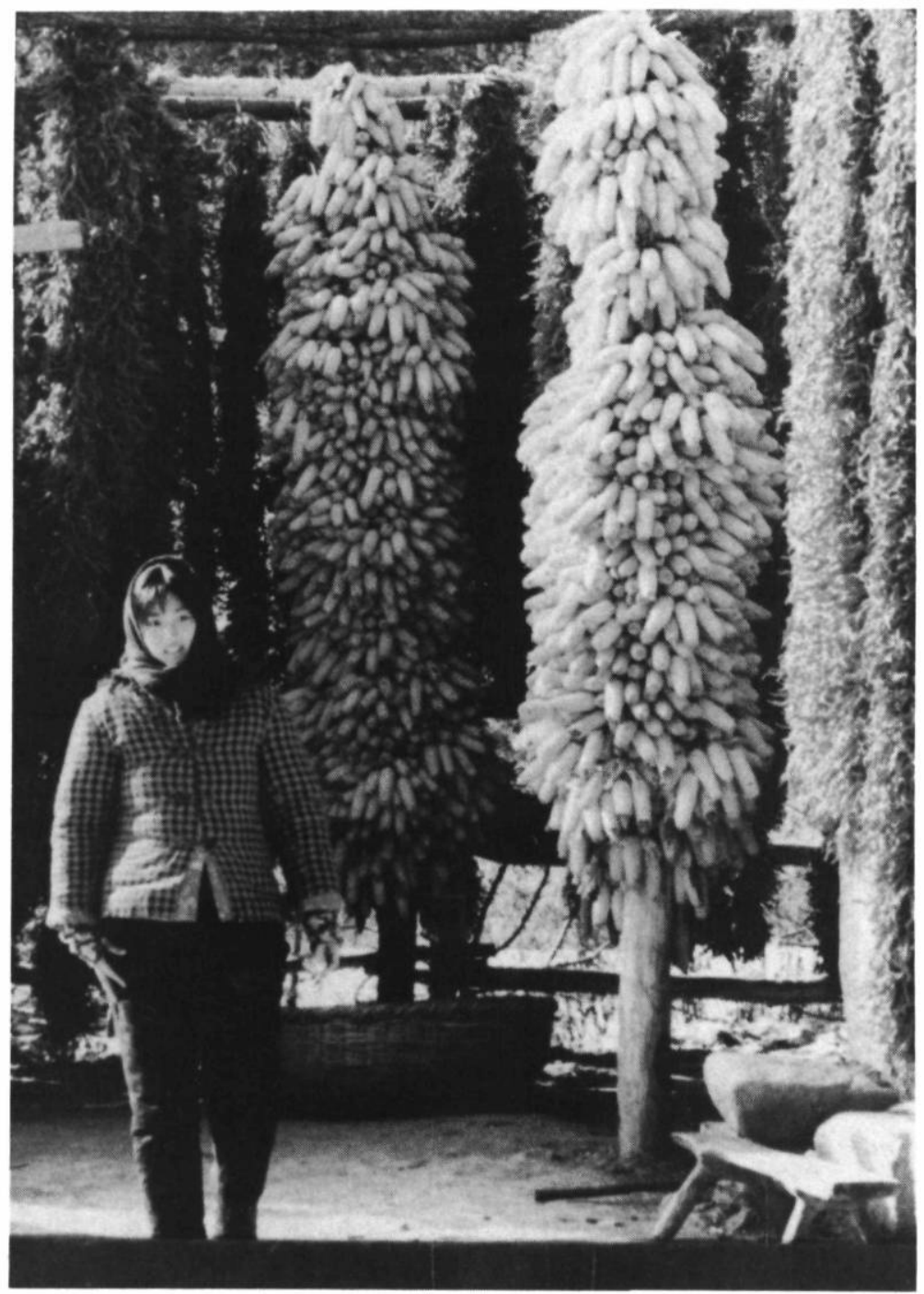

Quiju, une femme chinoise de Zhang Yimou (1992) 
En effet, de Sorgho rouge à Épouses et concubines, alcool et nourriture sont les symboles par excellence non seulement du pouvoir et du sexe, mais aussi du désir. Le navet à l'allure phallique dans Judou est le déclencheur du désir chez les amants; vers la fin du film, c'est encore l'acte de manger ce même légume bizarre qui les fait sourire et se séduire à nouveau. Dans Épouses et concubines, c'est lorsque la quatrième épouse refuse de prendre son repas avec ses «sœurs» que la maisonnée sait qu'elle se prépare à mener une lutte sans merci. Dans tous les films de Zhang, l'acte de consommer de la nourriture a lieu et occupe un temps considérable de la représentation. Il est, pourrait-on dire, plus présent que même les scènes de sexe et plus indicateur des sentiments d'amour, de haine, de jalousie. Lorsque Judou et Tianqing mangent ensemble, on les voit, en champ contre-champ, s'échanger des regards amoureux. Lorsqu'ils font l'amour, on ne voit que le regard éperdu de la femme.

Ainsi, malgré la profusion d'objets attrayants parce qu'inconnus, souvent nés de la fantaisie, c'est la nourriture qui lie et qui démarque le statut et la personnalité des individus. Symbole du pouvoir (ou de son exclusion), et signifiant du sexe (ou de son absence), la nourriture est le point d'ancrage du réel mais aussi de la fantaisie dans les films de Zhang.

\section{L'écriture : la quadrature du cercle?}

Je voudrais, en guise de conclusion, terminer en examinant la position de chacun face à ce qui est, sans doute, l'obsession chinoise la plus tenace, à savoir l'écriture. Cela permettra de déconstruire la forte dualité de mon texte. En effet, s'il est vrai que le cinéma de Chen comme de Zhang sont critiques de la culture chinoise dans son ensemble, alors l'écriture comme représentant du pouvoir doit y être signifiée. De fait, tous deux dénoncent la tyrannie de l'écriture mais tous deux demeurent néanmoins fascinés par les caractères chinois.

Chen, en bon lettré et philosophe, joue avec les caractères d'écriture. Dans Le Roi des enfants, il y a deux inventions. L'une est d'ordre étymologique : «Qu'est-ce que la vie? La vie, c'est comme son caractère d'écriture, huo : de l'eau à boire et une langue pour manger.» Cette invention étymologique montre une volonté de représenter la vie par quelque chose de concret, par quelque chose d'authentique, de moins élitiste. L'autre est de l'ordre de la pure invention : c'est un nouveau caractère, «buffle + urine», qui est un pied-de-nez aux lettrés d'antan (et d'aujourd'hui), si aliénés de la nature qu'ils sont incapables de conceptualiser cette association intime pour rendre le goût du buffle pour l'urine humaine. C'est, par ailleurs, cette image qui 
clôt le film Le Roi des enfants. Elle indique aussi la fantaisie, le besoin d'y mettre du sien, même dans le code écrit. Dans La Vie sur un fil, le bout de papier caché dans une cithare revêt une importance vitale pour le vieil aveugle qui croit qu'une prescription guérissant sa cécité y est inscrite. Le papier est vierge; néanmoins, le vieil aveugle refait le coup à son disciple qui, lui, remplacera ce papier tout blanc par un autoportrait de son amante. L'absence d'écriture dénote tout autant le caractère sacré de l'inscription que l'insensé. L'allusion à la culture basée sur l'écriture immuable qui transmet les mêmes messages depuis toujours ne saurait être écartée dans ces deux films.

Dans les films de Zhang, on ne badine pas avec l'écriture. L'écriture est un mur (comme les hommes) contre lequel on se bute. C'est l'inébranlable pouvoir patriarcal, ancestral. Dans Judou, la stèle funéraire, symbole phallique par exemple de ce même pouvoir, a le nom de Yang Jinshan, vénérable ancêtre, gravé sur une face; elle est placée, verticalement, dans l'entrejambes de son «fils» officiel, l'enfant illégitime de Judou et Tianqing, lui-même chevauchant un âne et observant ses deux parents rampant, en train de montrer leur piété filiale, en bloquant 49 fois la route au cortège funèbre.

Les protagonistes dans les films de Zhang, en général, ne savent pas lire. Ils sont donc exclus du monde écrit, codé. L'écriture n'est qu'un des signifiants du monde auquel ils n'appartiennent pas. Quiju, enceinte de 7 mois, doit se faire déchiffrer les verdicts de la cour par son entourage masculin. Les immenses portraits dans Épouses et concubines, qui surplombent la salle à manger, qui surveillent les épouses, ont une même valeur que les stèles ou registres des ancêtres. Tous les rituels non concoctés par les protagonistes féminins sont équivalents. Les paroles répétées plusieurs fois dans Judou et ensuite reprises ad nauseam dans Épouses et concubines sont tout aussi tyranniques : «Conformément aux lois anciennes des ancêtres» (an zuzong de lao guiju). La récurrence devient cause d'horreur. Elle énerve au point où l'on outrepasse sa condition. À la fin des films de Zhang, la femme meurt mais le cycle continue. Après avoir vu défiler, comme des points de ponctuation au long du film, les caractères pour «hiver», «été», «automne» et encore «hiver» - sans jamais de printemps - , l'«été» revient en un gros caractère pour marquer le recommencement, et la fin. L'effet esthétique est beau, mais (et) fait décrocher le spectateur.

La reprise, le cercle (vicieux) est tout aussi présent dans le cinéma de Chen et ce, à plusieurs niveaux : des scènes virtuellement identiques au début et à la fin de ses films 
(notamment le paysage du Yunnan dans Le Roi des enfants, la place Tiananmen dans La grande parade), mais aussi, plus localement, des histoires et des chansons sont récurrentes. On peut penser à la ritournelle, dans Le Roi des enfants («Sur une montagne, il y avait un temple; dans le temple, il y avait un moine qui chantait : qu'est-ce qu'il chantait? Sur une montagne, il y avait un temple; dans le temple, il y avait un moine qui chantait...»), qu'entonnent d'abord les «jeunes instruits» pour s'amuser, mais qui est vite reprise par les enfants. Dans ce même esprit, les films de Chen ne se terminent pas; ils recommencent.

Il y a donc des croisements, malgré la polarisation, dans les positions hanxu et yanggang. L'écriture est chez Chen comme chez Zhang, objet de savouration et de consommation. L'écriture demeure talismanique chez l'un comme chez l'autre; elle est à la fois fascinante et sert à la distanciation. L'écriture maintient ses trois types de pouvoir : magique, idéologique et culturel, peut-être en dépit des positions des cinéastes.

\section{Université de Montréal}

\section{NOTES}

1 Chen Kaige est le réalisteur de Terre jaune (Huang tudi), 1984; La grande parade (Da yue bing), 1985; Le Roi des enfants (Haizi wang), 1986, La Vie sur un fil (Bianzou bianchang), 1991 et Adieu à ma concubine (Bawang bieji), 1992. Il a également fait la scénarisation de plusieurs de ses propres films et les paroles des chansons de La Vie sur un fil.

Zhang Yimou est le directeur photo de Terre jaune, La grande parade, Le vieux puits (Laojing), 1987; il est l'acteur principal dans Le vieux puits et Guerrier de terre cuite (Qin Yong), 1990; il a réalisé Sorgho rouge (Hong Gaoliang), 1987; Code Name : Cougar (Daihao meizhou bao), 1988; Judou, 1989; Epouses et concubines (Dahong denglong gaogao gua), 1991 et Qiuju, une femme chinoise (Quiju da guansi), 1992.

Je n'ai pas vu le dernier film de Chen, Adieu, ni n'ai pu étudier Qiuju de Zhang. Aussi dois-je me limiter à leur filmographie jusqu'en 1991. Code Name: Cougar de Zhang n'est pas non plus à l'étude parce que ce film, fait pour des raisons commerciales, n'a rien à voir avec le cinéma de Zhang Yimou.

2 Cité par G. Barmé, Seeds of Fire (New York : The Moonday Press, 1989) p. 259. Le cinéaste utilise le même terme quand il est interviewé par Tony Rayns, dans son documentaire intitulé New Chinese Cinema (1987).

3 Entrevue menée par Paola Voci, à l'institut du cinéma de Beijing, 1990. Je tiens à remercier Dominique Brochu de m'avoir prêté l'enregistrement.

4 Tony Rayns, ibid.

5 Entrevue avec Paola Voci, ibid.

6 Idem. 
7 Tony Rayns, ibid.

8 Propos de Chen Kaige, au sujet de Terre jaune, lorsqu'interviewé par George Semsel (direction), Chinese Film: The State of the Art in the People's Republic (New York : Praeger, 1987) p. 82.

9 Dans une communication à la Troisième Conférence internationale de I'Asian Cinema Studies Society, en juin 1992, j'ai présenté une lecture des films de Zhang Yimou d'après les événements de sa vie personnelle. Cette perspective permet d'expliquer la progression vers un pessimisme de plus en plus marqué dans ses films, justement à cause des contraintes de divers ordres auxquelles Zhang fait face. Bien sûr, cette lecture n'en est qu'une parmi d'autres, notamment celle de l'allégorie politique, sans conteste la plus usuelle.

10 C'est ainsi que François Jullien traduit le terme dans son livre Éloge de la fadeur (Arles, Paris : Picquier, 1991). Le travail de F. Jullien sur l'esthétique chinoise fournit un éventail d'illustrations de ce mode hanxu qu'il appelle «allusif» pour la poésie ou «fade» pour la peinture, et encore beaucoup d'autres termes comme «détachement», «savouration». Voir La Valeur allusive (École française d'Extrême-Orient, 1985) et tout particulièrement dans Éloge de la fadeur, le chapitre 14 : «La fadeur ou la force.»

11 À l'exception du travail de Xu Fuguan qui, dans les années 60 et 70 , reprenait le vocabulaire esthético-philosophique traditionnel, et y discernait non seulement une seule notion du beau (combinant les pôles yin et yang), mais bien deux types, l'un yin et l'autre yang. Voir Xu Fuguan, Zhongguo yishu jingshen (L'Esprit de l'art chinois) (Shenyang : Chunfeng wenyi chubanshe, 1987) pp. 138-156.

12 Zhang Lihui, «A Cross-Cultural Study of Red Sorghum and Onibaba», communication présentée à la Troisième Conférence internationale de l'Asian Cinema Studies Society, 14 juin 1992. Elle y affirmait que Zhang Yimou avait fait visionner Onibaba à son équipe avant de tourner Sorgho rouge, même si, par la suite, il avait nié toute influence non chinoise.

13 Je tiens à remercier Lü Tonglin qui m'a signalé et procuré ce texte.

14 C'est ce qui ressort de l'analyse de Lin Niantong, dans «Zhongguo dianying lilun yanjiu zhong youguan gudian meixue wenti de tantao", Youjing (1985) pp. 30-56. Lin y discute du rapport entre l'esthétique de la tradition picturale chinoise et le cinéma en tant qu'art. Il situe ce rapprochement dans le cinéma des années 40 et la théorisation qui fut développée à ce sujet au début des années 60.

15 Jenaro Talens, «Une histoire alternative du cinéma», séminaire donné au Département de littérature comparée de l'Université de Montréal à l'hiver 1990.

16 Dossier de presse, Era International, Taiwan, 1991, distribué par Alliance / Viva Film, Montréal.

\section{OUVRAGES CITÉS}

Anderson, Eugene. Food in Chinese Culture : Anthropological and Historical Perspectives. K.C. Chang, éd., New Haven \& London : Yale UP, 1977.

Aubert, J.P. «La Cinquième génération: Chen Kaige». Cahiers du cinéma (avril 1988) pp. 49-51.

Barmé, G. \& J. Minford. Seeds of Fire. New York : The Noonday Press, 1989. 
Can Xue. «Tuwei biaoyan» (Performance rompant l'encerclement). Shanghai: Shanghai wenyi chubanshe, 1990. Communication présentée à la Maison d'édition des Arts et Lettres de Shanghai, s.d., publiée à cette même maison d'édition, Shanghai wenyi chuban, 1990.

Chua, Lawrence. «Making Movies (or Trying to) in China». Premiere (mars 1992) pp.29-30.

Hua Jun. Di wudai daoyan (Les Réalisateurs de la Cinquième génération). Beijing : Xiandairen congshu, 1988.

Jullien, François. La Valeur allusive. École française d'Extrême-Orient, 1985. Jullien, François. Éloge de la fadeur. Arles, Paris : Picquier, 1991.

Li Tong, «Hong gaoliang xixing», Wenxing (Taipei) 119 (mai 1988) pp. 4048 .

Lin Niantong. "Zhonguo dianying lilun yanjiu zhong youguan gudian meixue wenti de tantao». Youjing. Hong Kong : Suye chubanshe, 1985, pp. $30-56$.

Pan, Lynn. "A Chinese Master». The New York Times Magazine (1er mars 1992) pp. 30-38.

Schell, Orville. «Back to China laden with New Ideas». Dossier de Presse pour La Vie sur un fil (distribution Aska Films) s.p.

Semsel, George S. (direction). Chinese Film : The State of the Art in the People's Republic. New York : Praeger, 1987.

Xu Fuguan. Zhongguo yishu jingshen (L'Esprit de l'art chinois). Shenyang : Chunfeng wenyi chubanshe, 1987.

Zhang Yimou. «Gaoliang di de chuanshuo». Wenxing (Taipei) 119 (mai 1988).

Zhong Chenxiang. "Dianying yuyan xiandaihua tansuo de jiejin: ping yingpian Honggaoliang». Renmin ribao (mars 1988).

Zhou Yan. «Xinchao meishu de yuyan xingtai», Meishu (1988) pp.132-144.

\section{GLOSSAIRE DES PRINCIPAUX TERMES CHINOIS}

(par ordre alphabétique)

an zuzong de lao guiju

Bawang bieji

Bianzou bianchang

Chen Kaige

Da hong denglong gaogao gua

Da yuebing

Daihao meizhou bao

Haizi wang

hanxu

Hong gaoliang

Huang tudi

按祖宗的老规矩

霸王別姬

邊走邊唱

堜凱歌

大紅燈籠高高掛

大閲兵

代號美洲豹

孩子王

含蓄

紅高粱

黄土地 
huo

Judou

Laojing

pingdan

Qinyong

Qiuju da guansi

sanjue

wenyi zai dao

wo kan bu jian

Xu Fuguan

yang

yanggang

yanggang yinrou zhi mei

yin

Zhang Yimou

ziwo pengzhang
活

粷豆

老井

平淡

秦俑

秋料打官司

三絶

文謷载道

我看不見

徐復觀

陽

陽剛

陽剛㓌柔之美

院

張㢣謀

自我膨脹 